\title{
Preparation of Anisole in Phenol by Zn/HZSM-5 Catalyst from Walnut Shell Catalyzed Liquefaction
}

\author{
Jianxiang Liu, ${ }^{\text {a,b,d }}$ Yuhan Lu, ${ }^{\text {a,b,c }}$ Ruiling Wang, ${ }^{\text {e }}$ Yuanbo Huang, Can Liu, ${ }^{\text {a,b,c,f }}$ and \\ Zhifeng Zheng a,b,d,f,*
}

The promotional effect of $\mathrm{Zn}$ was investigated relative to the calcining activation of HZSM-5. It was found that the lignin separated from walnut shell could be liquefied into small molecular ethers such as anisole by the promotional effect of the modified catalyst. Experimental results showed that, after the loading of $\mathrm{Zn}^{+}$, the HZSM-5 catalyst retained its original crystal structure, which exhibited good metal dispersion. Besides, due to the existence of two interactions respectively of $\mathrm{Zn}$ with $\mathrm{Al}$ and $\mathrm{Zn}$ with a Brønsted-Lowry acid on the Zn/HZSM-5 catalyst surface, the remaining catalyst intergranular distance increased, particularly for the $2 \%$ $\mathrm{Zn} / \mathrm{HZSM}-5$ catalyst. The inside of the catalyst was in a state of spherical particle aggregation with a narrow pore size distribution and uniform particle size. The peak intensity of the Si-O stretching vibration was affected by the content of $\mathrm{Zn}^{+}$, which was least affected for the $2 \%$ $\mathrm{Zn} / \mathrm{HZSM}-5$ catalyst. The signal peak of this small molecule ether was not found in the absence of the metal ions, and the catalytic effect of $2 \%$ $\mathrm{Zn} / \mathrm{HZSM}-5$ was more distinct. Calculated by the mass difference method, the yield of the liquid via the $2 \% \mathrm{Zn} / \mathrm{HZSM}-5$ catalyst was $59.8 \%$, including phenol and small molecule ethers represented by anisole.

Keywords: Anisole; Catalyzed liquefaction; Walnut shell; Lignin; Catalysts

Contact information: a: National-Local Joint Engineering Research Center for Highly-Efficient Utilization Technology of Forest Biomass Resources, Southwest Forestry University, Kunming 650224 China; b: Key Laboratory for Highly-Efficient Utilization of Forest Biomass Resources in the Southwest China, National Forestry and Grassland Administration, Yunnan Provincial International Joint Research Center for Bioenergy, Southwest Forestry University, Kunming 650224 China; c: School of Materials Science and Engineering, Southwest Forestry University, Kunming 650224 China; d: School of Chemical Engineering, Southwest Forestry University, Kunming 650224 China; e: School of Forestry, Southwest Forestry University, Kunming 650224 China; f: Fujian Provincial Engineering and Research Center of Clean and High-Valued Technologies for Biomass, Xiamen Key Laboratory for High-Valued Conversion Technology of Agricultural Biomass College of Energy, Xiamen University, Xiamen 361102 China;

*Corresponding author: zhifeng.zheng@xmu.edu.cn

\section{INTRODUCTION}

The environmental impact of global warming is motivating a shift to biomass resources, which entail less environmental contamination and fewer health risks compared with fossil fuel (Isa et al. 2018). Among the bio-renewable polymers, lignin, as the second most ample biomass next to cellulose, is an amorphous and carbon-rich aromatic polymer composed with aromatic structure units (Kang et al. 2013). The structure of lignin is based on phenylpropanoid units involving alkyl aryl ether linkages, some of which carry phenolic hydroxyl groups (Cox 2012). Native lignin has complex and three-dimensional amorphous structures, which limits the commercial utilization of it that less than $5 \%$ of the lignin removed from wood during pulping operations (Alzari et al. 1990). Due to environmental 
concerns and the shortage of petroleum resources, lignin has been investigated to be a lower-cost raw material compared to other current organic materials (Kubo and Kadla 2005; Gordobil et al. 2014; Mota et al. 2016). The prominent properties of lignin, such as high abundance, low weight, and environmental friendliness, as well as its antioxidant, antimicrobial, and biodegradable nature, make it an ideal candidate for the development of novel polymer composite materials (Thakur et al. 2014). The prerequisite to synthesize a novel polymer composite material is that the raw materials must be small molecules with definite molecular structures, which means that the liquefaction of lignin is essential for such syntheses (Lee et al. 2011; Cinelli et al. 2013; Ma et al. 2016). The preparation of small-molecule platform compounds by liquefying pure lignin or other biomass with high lignin contents is also a new direction for biomass research and utilization. Walnut shell is a widely available biomass resource having lignin contents of that can reach up to $56.6 \%$ (Zheng et al. 2007). It can be concluded from previous studies that phenol is a good liquefaction agent for walnut shell, because that it can degrade walnut shell to produce small molecules by providing an active group for the liquefaction (Yin et al. 2012).

Catalysts have played a crucial role in the utilization of biomass in recent years, especially in the liquefaction of biomass. The zeolite catalyst is widely applied due to its convenience of being readily separated and reused without corrosion (Batistela et al. 2017). A crystalline aluminosilicate zeolite (molecular sieve) catalyst (HZSM-5) is universally used for the catalytic conversion of olefins (Sadrameli 2016), and it also can be applied for the preparation of some chemicals by various supported modifiers (Li et al. 2015; Xu et al. 2015; Fattahi et al. 2016; Shen et al. 2016). Several papers had reported the use of HZSM5 zeolite for the catalyzed fast pyrolysis of lignin. For instance, Jackson et al. (2009) screened various catalysts, including HZSM-5, KZSM-5, Al-MCM-41, solid phosphoric acid, and a commercial hydrodesulfurization catalyst $\left(\mathrm{Co} / \mathrm{Mo} / \mathrm{Al}_{2} \mathrm{O}_{3}\right)$ in oxygenated form, and concluded that the HZSM-5 zeolite was the best choice for producing deoxygenated organics. Mullen et al. (2017) studied the catalytic fast pyrolysis of lignin from four different sources by using both $\mathrm{HZSM}-5$ and $\mathrm{CoO} / \mathrm{MoO}_{3}$ in a microscale pyroprobe, and the former was proved to be more efficient than the later to produce oxygen-free aromatics, regardless of the types of lignin. Asadieraghi et al. (2015) studied the upgrading of pyrolysis vapors derived from palm kernel shells catalyzed by various catalysts, including the HZSM-5 and Ga/HZSM-5, the main products obtained by which were alkoxy phenol and aromatic hydrocarbons. HZSM-5 exhibited better activity in the aromatization and deoxygenation during the upgrading of pyrolytic vapors, although it did decrease the biooil yield. Compared with that, the Ga/HZSM-5 catalyst increased the bio-oil yield, and the aromatics selectivity can be enhanced by the introduction of appropriate amount of gallium. Huang et al. (2017) explored inexpensive catalysts supported with Ni/HZSM-5 for the depolymerization of kraft lignin with formic acid at lower temperatures. Results showed that the presence of a supported metal catalyst can effectively reduce the molecular weight (MW) of the depolymerized lignin product, although the catalyst did not have effects on the yield of product. On the other hand, the Zn/HZSM-5 catalyst can be used for both the catalytic conversion of methanol to aromatics and the aromatization of alkanes (Abdelsayed et al. 2015; Fei et al. 2015; Lai and Veser 2016); the content of Zn markedly enhances the aromatization performance (Xin et al. 2013). Zhou et al. (2016) studied the effect of temperature on the catalyst HZSM-5 for the pyrolysis vapor derived from the upgrading of lignin. Results showed that a high catalyst temperature $\left(600^{\circ} \mathrm{C}\right)$ was required to produce oxygen-free aromatics, and an organic liquid product containing $70 \mathrm{wt} \%$ of oxygen-free aromatics (mainly benzene and toluene) was obtained at $600{ }^{\circ} \mathrm{C}$. 
Anisole is not only used as a solvent, but also used in the preparation of essential oils and organic synthesis (Jaworski et al. 2005). It can be obtained by reacting dimethylsulfate with phenol in an alkaline solution, but the dimethylsulfate is highly toxic. Current research is aimed at finding a healthier, simpler, and lower-cost preparation method to obtain anisole. In this paper, the $\mathrm{Zn} / \mathrm{HZSM}-5$ catalyst is used to catalyze the liquefaction of walnut shell biomass, and anisole is obtained via catalyzed liquefaction at a low temperature $\left(150^{\circ} \mathrm{C}\right)$ and a low atmospheric pressure.

\section{EXPERIMENTAL}

\section{Materials}

Walnut shell powder was provided by Dali Yangbi Walnuts Co., Ltd. (Dali, China). The powder was less than $74 \mu \mathrm{m}$ in size. The lignin content of walnut shell is known to contain many phenolic hydroxyl groups, which are capable of reacting with formaldehyde to produce long-chain substances. Phenol was purchased from Tianjin Fengchuan Chemical Reagent Technology Co., Ltd. (Tianjing, China). The catalyst HZSM-5 was obtained from Tianjin Nanhua Catalyst Co., Ltd. (Tinjing, China). The silicon-to-aluminum ratio $(\mathrm{Si} / \mathrm{Al})$ of HZSM-5 was 38. After the activation reaction at $550{ }^{\circ} \mathrm{C}$ for $5 \mathrm{~h}$, the pore size increased to $6.93 \mathrm{~nm}$ (the non-activated diameter was $3.83 \mathrm{~nm}$ ). Zinc chloride was purchased from Xilong Chemical Co., Ltd. (Guangdong, China). Concentrated sulfuric acid was obtained from the Yunnan Yanglin Industrial Development Zone, Yunnan Pharmaceutical Co., Ltd. (Yunnan Sheng, China). All reagents were of analytical grade.

\section{Catalyst synthesis}

The HZSM-5 was treated by activation at $550{ }^{\circ} \mathrm{C}$ for $5 \mathrm{~h}$ to be used as support material. The $\mathrm{Zn} / \mathrm{HZSM}-5$ catalysts with different mass ratios of $\mathrm{ZnCl}_{2}$ loading ( $1 \%, 1.5 \%$, $2 \%, 2.5 \%$, and $3 \%$ ) were prepared using the wet impregnation method, as shown in Fig. 1.

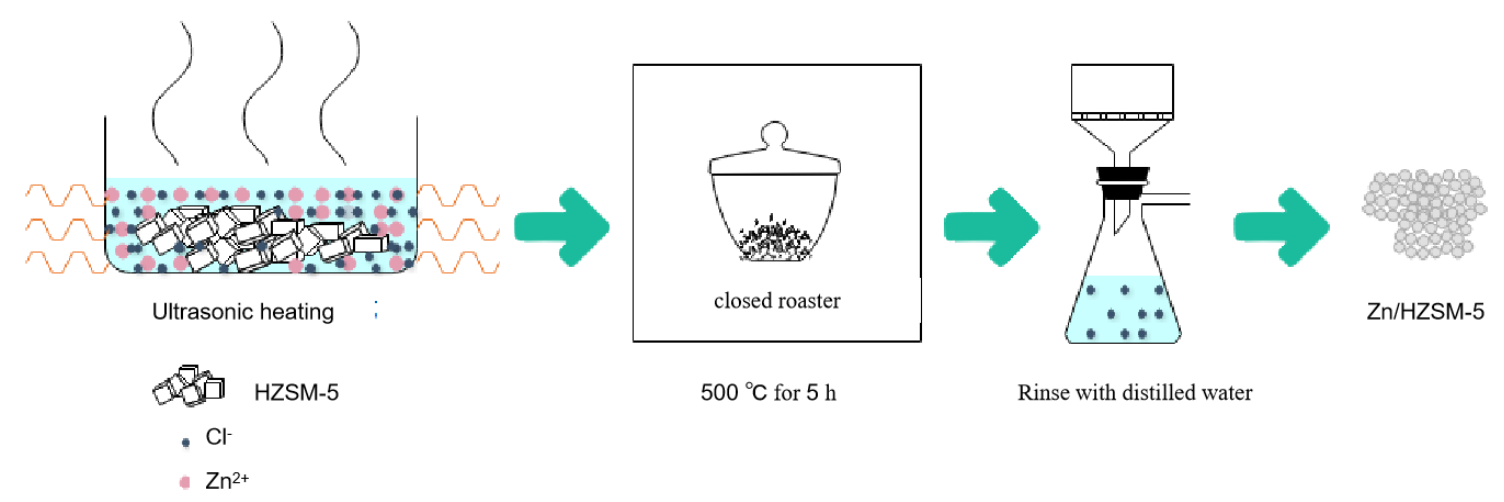

Fig. 1. Synthetic process of the Zn/HZSM-5 catalyst

\section{Catalytic liquefied walnut shell}

The walnut shell powder was first mixed with phenol with a mass ratio of 1:5 (powder to phenol), and Zn/HZSM-5 was subsequently added, with a mass of 3\% of phenol. The reaction lasted for $2.5 \mathrm{~h}$ at $150{ }^{\circ} \mathrm{C}$ under atmospheric pressure. After that, the solution was filtered while it was still hot. Afterwards, the filtrate was kept for subsequent reactions. The process of catalytic liquefied walnut shells is shown in Fig. 2. 


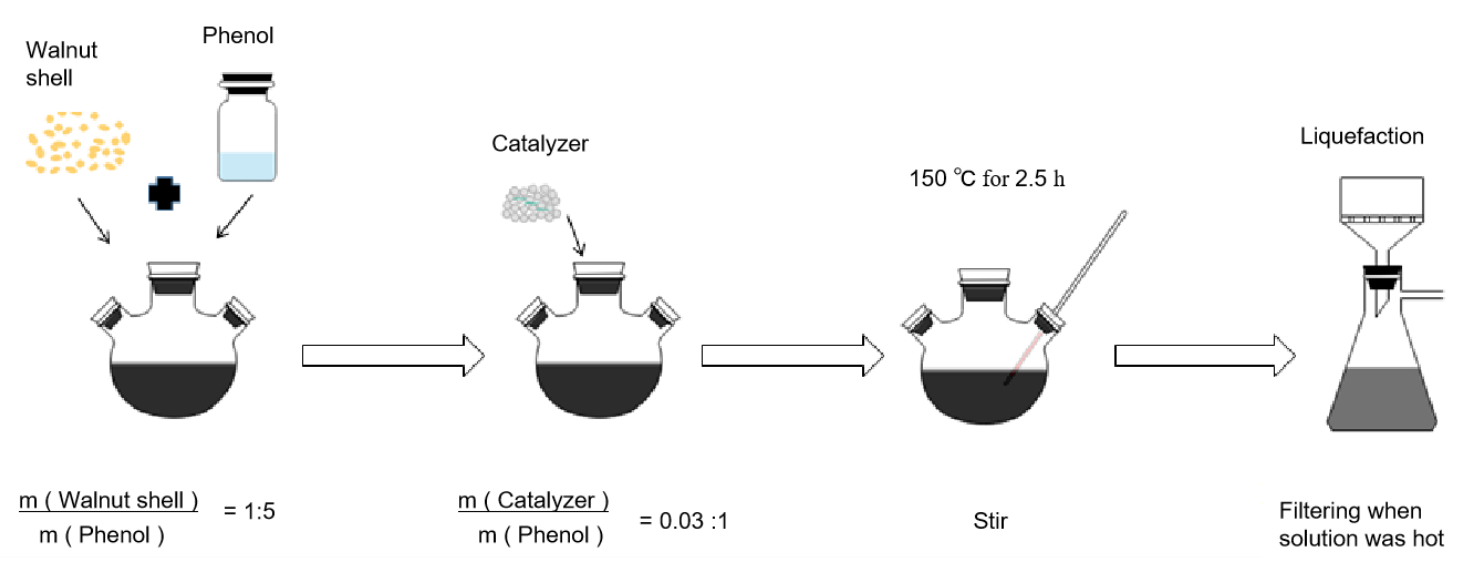

Fig. 2. Catalyzed liquefaction of walnut shells in phenol via Zn/HZSM-5 catalyst

\section{Methods}

Characterization of catalysts

X-ray diffraction analysis (XRD) was performed on an Ultima IV X-ray diffractometer (KYOWAGLAS-XA; Rigaku, Kuraray, Japan), utilizing the Si holder and scanning from $5^{\circ}$ to $35^{\circ}(2 \theta)$. Nitrogen adsorption experiments (BET measurement method) were performed with an ASAP 2020 plus HD 88 instrument (Micromeritics, Shanghai, China). The Fourier transform infrared (FTIR) analysis was performed with a Nicolet 6700 FTIR spectrometer (Thermo Fisher Scientific, Waltham, MA, USA) using the KBr pellets.

\section{Characterization of liquefied products}

The carbon-13 nuclear magnetic resonance $\left({ }^{13} \mathrm{C}-\mathrm{NMR}\right)$ analysis was performed on an AVANCE III $600 \mathrm{MHz}$ NMR spectrometer (Bruker, Billerica, MA, USA) at $298 \mathrm{~K}$ and at a frequency of $150 \mathrm{MHz}$. Deuterated methanol was used as the solvent. Gas chromatography-mass spectrometry analysis (GCMS) was performed using a gas chromatograph-mass spectrometer (GCMS-QP2010 SE; Shimadzu Corp., Kyoto, Japan) utilizing chromatographic-grade methanol as a solvent. The FTIR analysis was performed with a Nicolet 6700 FTIR spectrometer (Thermo Fisher Scientific, Waltham, MA, USA) using the $\mathrm{KBr}$ pellet method.

\section{RESULTS AND DISCUSSION}

\section{Characterization of Catalysts \\ XRD analysis}

The XRD patterns for the prepared HZSM-5 catalysts loading Zn are shown in Fig. 1a. The high intensity diffraction peak between approximately $2 \theta=20^{\circ}$ to $25^{\circ}$ indicated that all the catalysts retained their HZSM- 5 crystal structure after being loaded and calcined with different metal oxides (Abdelsayed et al. 2015). The absence of any metal oxide peaks in the XRD patterns showed there was a good metal dispersion with a small particle size on the zeolite surfaces (Abdelsayed et al. 2013). The small angle XRD patterns of the microporous materials were sensitive to the presence of any particles inside their microporous channel structures, where the intensity and the d-spacing of the diffraction peak changed accordingly (Wang et al. 2017). To compare the XRD patterns of these catalysts, the data were normalized at $2 \theta=23^{\circ}$. Figure $1 \mathrm{~b}$ shows the influence of metal 
loading on the microporous structure monitored at low angle diffraction peaks between approximately $2 \theta=7^{\circ}$ to $10^{\circ}$ when the data were normalized. The value of the low angle diffraction peaks between approximately $2 \theta=7^{\circ}$ to $10^{\circ}$ was noticeably less than that of pure HZSM-5 catalysts except for the $2 \%$ ZnHZSM-5. According to the equation for Bragg's Law $(2 d \times \sin \times \theta=n \lambda)$, a smaller angle indirectly reflects an increase in the interplanar spacing. This could have been due to a strong interaction between the $\mathrm{Zn}$ and the $\mathrm{Al}$ framework on the catalyst surface, which led to a lower crystallinity (Abdelsayed et al. 2013). Additionally, there was a strong interaction between the $\mathrm{Zn}$ and the external Brønsted-Lowry acid sites on the external surface of the catalyst (Mhamdi et al. 2009). There was some balance between the two interactions using $2 \% \mathrm{Zn}$.

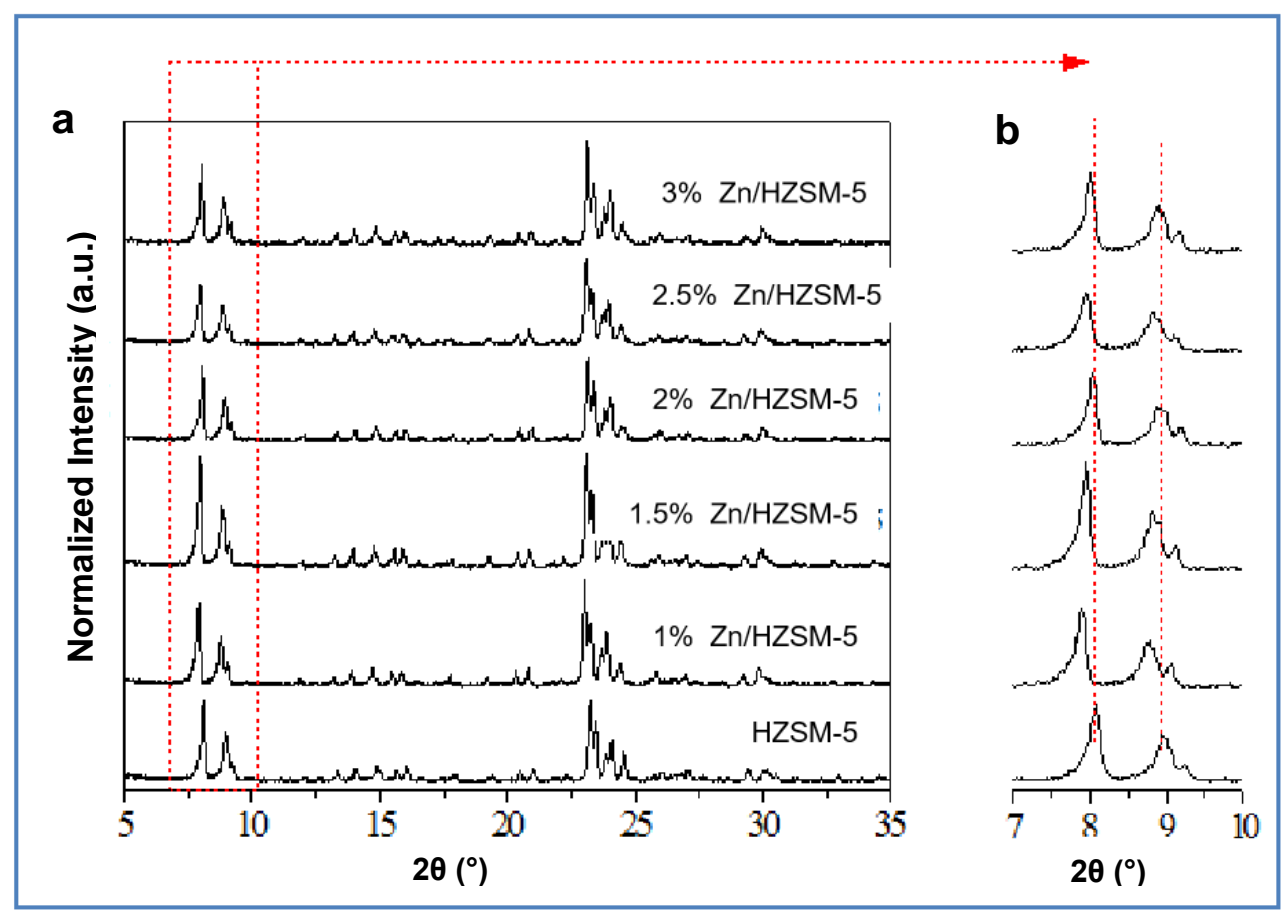

Fig. 3. (a) XRD patterns for freshly calcined HZSM-5 supported with different proportions of Zn catalysts and (b) small angle XRD patterns for these catalysts

\section{Surface area and micropore analysis}

The concentration of $\mathrm{ZnCl}_{2}$ and HZSM-5, the average pore size $\left(P_{\text {ave }}\right)$, and the Brunauer-Emmett-Teller (BET) surface area $\left(S_{\mathrm{BET}}\right)$ of the studied catalysts are listed in Table 1. The aperture distribution diagrams for the Zn/HZSM-5 catalysts are shown in Fig. 4b. As shown, the increase of $\mathrm{Zn}$ load resulted in the trend of both increasing and decreasing of pore size. When the $\mathrm{Zn}$ load was $2 \%$, the pore size reached maximum, and the pore size of the catalyst loaded was still less than the unloaded catalyst. The specific surface area slowly declined before suddenly declining further, with an abrupt turning point of $2.5 \%$, which was mainly due to the strong interaction between $\mathrm{Zn}$ and the external Brønsted-Lowry acid sites that blocked some of the pore structures on the external surface of the catalyst (Mhamdi et al. 2009). When the diameter was closer to that of the original HZSM-5 catalyst, the interaction between Zn and external Brønsted-Lowry acid sites on the catalyst was lower, e.g., the 2\% Zn/HZSM-5 catalyst (Wang et al. 2016), consistent with the similar $d$-spacing shift observed in the XRD data. 
Table 1. Properties of Prepared Zn/HZSM-5 Catalysts

\begin{tabular}{|c|c|c|c|c|c|}
\hline No. & Samples & $\begin{array}{c}\text { Concentration } \\
\text { of } \mathrm{ZnCl}_{2}(\mathrm{wt} \%)\end{array}$ & $\begin{array}{c}\text { Concentration of } \\
\mathrm{HZSM}-5(\mathrm{wt} \%)\end{array}$ & $\begin{array}{c}\text { Average Pore } \\
\text { Size }(\mathrm{nm})\end{array}$ & $\begin{array}{c}\text { BET Surface } \\
\text { Area }\left(\mathrm{m}^{2} / \mathrm{g}\right)\end{array}$ \\
\hline 1 & $\mathrm{HZSM}-5$ & 0 & 100 & 7.0489 & 293.1314 \\
\hline 2 & $1 \% \mathrm{Zn} / \mathrm{HZSM}-5$ & 1 & 99 & 6.6478 & 274.7728 \\
\hline 3 & $1.5 \% \mathrm{Zn} / \mathrm{HZSM}-5$ & 1.5 & 98.5 & 6.7797 & 275.7754 \\
\hline 4 & $2 \% \mathrm{Zn} / \mathrm{HZSM}-5$ & 2 & 98 & 6.9337 & 260.2011 \\
\hline 5 & $2.5 \% \mathrm{Zn} / \mathrm{HZSM}-5$ & 2.5 & 97.5 & 6.8840 & 268.7894 \\
\hline 6 & $3 \% \mathrm{Zn} / \mathrm{HZSM}-5$ & 3 & 97 & 6.6921 & 190.4880 \\
\hline
\end{tabular}
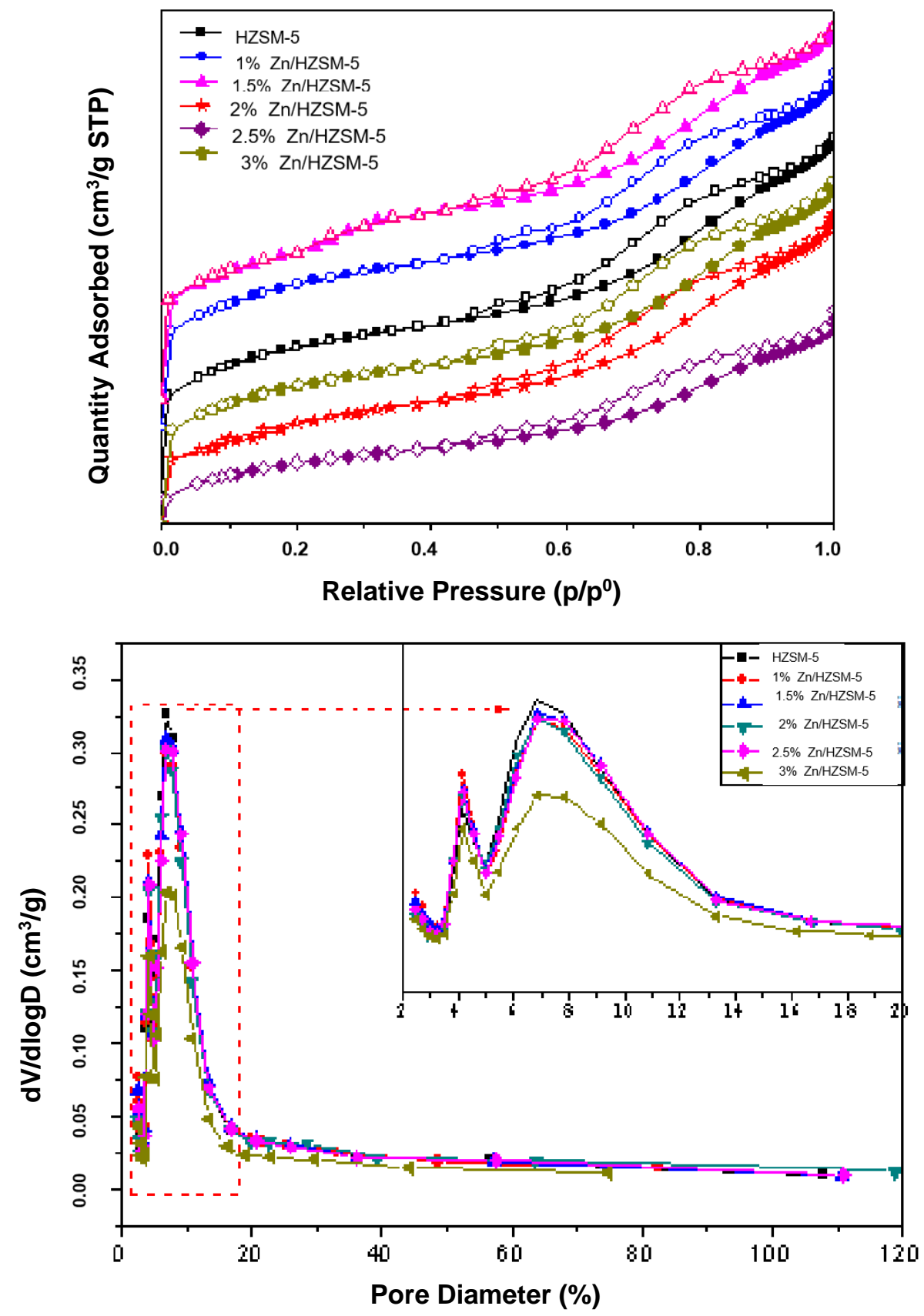

Fig. 4. (a) $\mathrm{N}_{2}$-adsorption isotherms and $\mathrm{N}_{2}$-desorption isotherms for $\mathrm{Zn} / \mathrm{HZSM}-5$ catalysts. The solid and open symbols are for the $\mathrm{N}_{2}$-adsorption and $\mathrm{N}_{2}$-desorption points, respectively. (b) Aperture distribution diagram for $\mathrm{Zn} / \mathrm{HZSM}-5$ catalysts 
The $\mathrm{N}_{2}$-adsorption isotherms, $\mathrm{N}_{2}$-desorption isotherms, and the aperture distribution diagrams for the Zn/HZSM-5 catalysts are shown in Fig. 4a. All catalysts exhibited typical type IV isotherms with a small hysteresis loop in the range of $p / p_{0}=0.4$ to 0.9 , which revealed the existence of mesoporous in the zeolites catalysts. This was due to the adsorption of $\mathrm{N}_{2}$ on the external surface of their crystallites and the capillary condensation in the spaces between the crystallites (Liu et al. 2012). All of the small hysteresis loops belong to the H1 type, according to the new International Union of Pure and Applied Chemistry (IUPAC) classification (Ihsan et al. 2016). This showed that the inside of catalysts was still in a state of spherical particle aggregation with a narrow pore size distribution and uniform particle size.

\section{FTIR analysis of catalysts}

In most cases, infrared spectrum are mainly used to analyze organic functional groups but not inorganic matter. However, the use of infrared spectroscopy to analyze the oxides of inorganic materials, such as catalysts, does exist. The type of cation for inorganic materials can affect the vibrational frequency of its anion (Davantès et al. 2015). The influence of $\mathrm{Zn}^{+}$on the whole catalyst system was analyzed by noting the frequency of its infrared vibration. As shown in Fig. 5, with the addition of $\mathrm{Zn}^{+}$, the amplitude of the Si-O bond stretching vibration first showed a downward trend after rising at $1092 \mathrm{~cm}^{-1}$, which was the smallest for $2 \% \mathrm{Zn} / \mathrm{HZSM}-5$, indicating a positive impact after the negative impact of that along with the $\mathrm{Zn}^{+}$addition. The reason for this was that a small amount of $\mathrm{Zn}^{+}$ entered the pores of the catalyst, due to the strong interaction between the $\mathrm{Zn}$ and external Brønsted acid sites on the catalyst external surface that blocks some of its pore structures (Wang et al. 2016). That affected the stretching vibration absorption peak of the Si-O bond. After reaching the critical point ( $\% \mathrm{Zn} / \mathrm{HZSM}-5)$, the added $\mathrm{Zn}^{+}$expanded this impact (Pradeep and Chandrasekaran 2006).

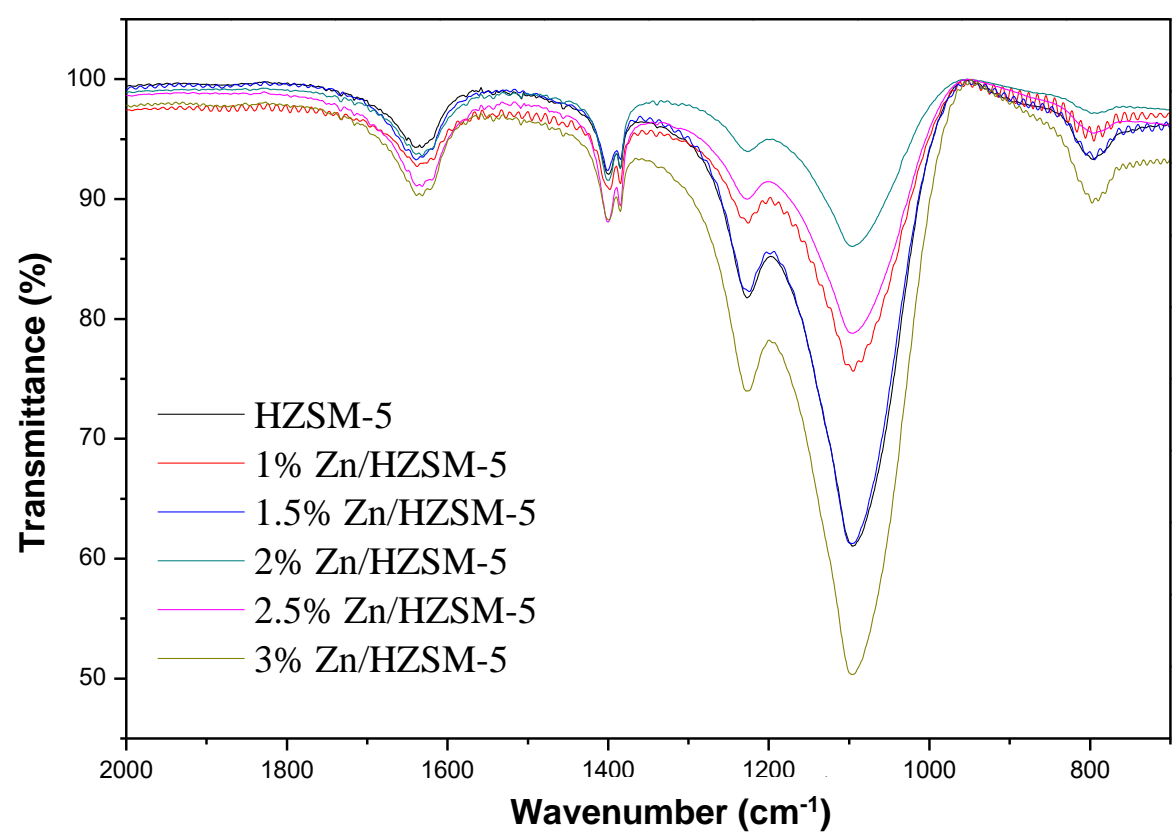

Fig. 5. FTIR spectra for calcined HZSM-5 supported with different proportions of Zn catalysts 


\section{Characterization of Liquefied Products}

\section{NMR analysis}

The ${ }^{13} \mathrm{C}$-NMR spectrum of the liquefied product is shown in Fig. 6. The analysis of the liquefied material catalyzed through the $2 \% \mathrm{Zn} / \mathrm{HZSM}-5$ catalyst and the catalyst-free liquefied material was conducted because the NMR spectrum of the catalyst was basically the same in this work, according to the data. The region inspected was the signal peak of the solvent phenol in the catalytic liquefaction process from about $115 \mathrm{ppm}$ to $156 \mathrm{ppm}$. Within this region, the peak at 156.04 ppm corresponded to the carbon atom at position 1 where the phenolic hydroxyl group was located; the peak at $115.94 \mathrm{ppm}$ was related to the ortho position respectively at positions 2 and 6; the peak at $130.32 \mathrm{ppm}$ was characteristic of positions 3 and 5; and the signal peak at $121.18 \mathrm{ppm}$ was related to position 4 of the para-phenolic hydroxyl group (Park and Riedl 2000). The signal peak near 50 ppm was related to the carbon atom at position 7 of methoxytoluene, but it did not appear in the catalyst-free liquefaction product. The presence of catalyst promoted formation of methoxytoluene.

\section{a}
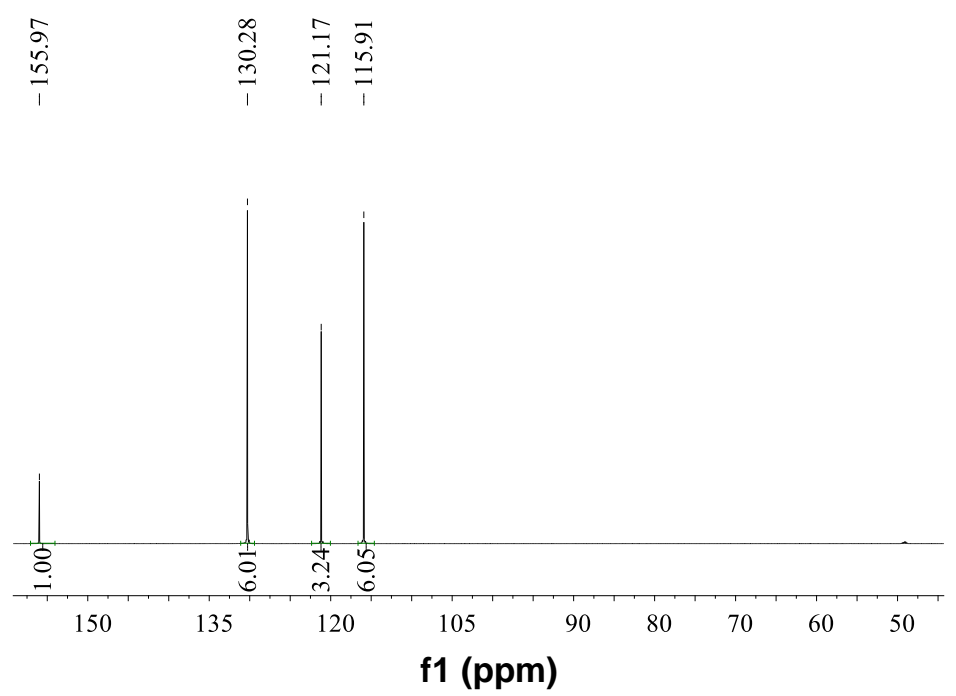

b

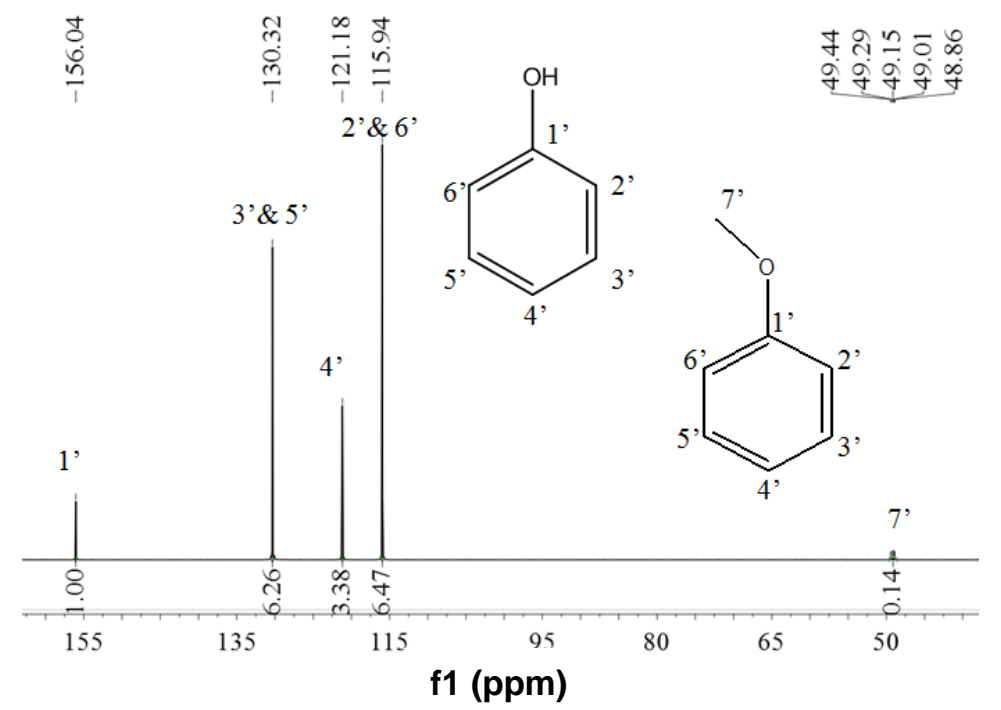

Fig. 6. (a) NMR spectra for catalytic liquefaction products with $2 \% \mathrm{Zn} / \mathrm{HZSM}-5$ catalysts and (b) NMR spectra for catalytic liquefaction products with the pure catalyst 


\section{GC-MS analysis}

Figure 7 shows the GC-MS spectrum of the liquefied product. As shown in Fig. 7 , after the addition of the catalyst, the liquefied product had a higher content of methoxytoluene. However, the content of the substance in the liquefied material with the customary catalyst was not conspicuous. The content of methoxytoluene was the highest in the liquefied products catalyzed by $2 \% \mathrm{Zn} / \mathrm{HZSM}-5$, while the other products were relatively lower. It was presumed that the catalytic effect was remarkable because the crystal structure of the $2 \% \mathrm{Zn} / \mathrm{HZSM}-5$ catalyst had just reached the saturation point, and the catalytic effect was remarkably reduced due to the cluster phenomenon that was caused by an excess of $\mathrm{Zn}$ higher than 2\% (Berry and Smirnov 2009).

\section{FTIR analysis of liquefied product via Zn/HZSM-5 catalysts}

Infrared spectroscopy has been shown to be a highly effective way to investigate specific interactions between polymers. Fourier transform infrared analysis can be used to qualitatively and quantitatively study the mechanism of interpolymer miscibility through hydrogen-bonding (Kadla and Kubo 2004). Figure 8 shows the infrared spectra of the liquefied products obtained via the catalytic liquefaction by the catalysts. As shown in Fig. 8 , the signal peak of the macromolecule ether bond appeared in the liquefied product with the HZSM-5 catalyst at $1040 \mathrm{~cm}^{-1}$, but the addition of the catalyst liquefied infrared spectrum did not find the same signal peak. Instead, a small molecular ether bond signal peak appeared at $1061 \mathrm{~cm}^{-1}$, indicating that the catalyst promoted the degradation of macromolecules (Kordić et al. 2017).

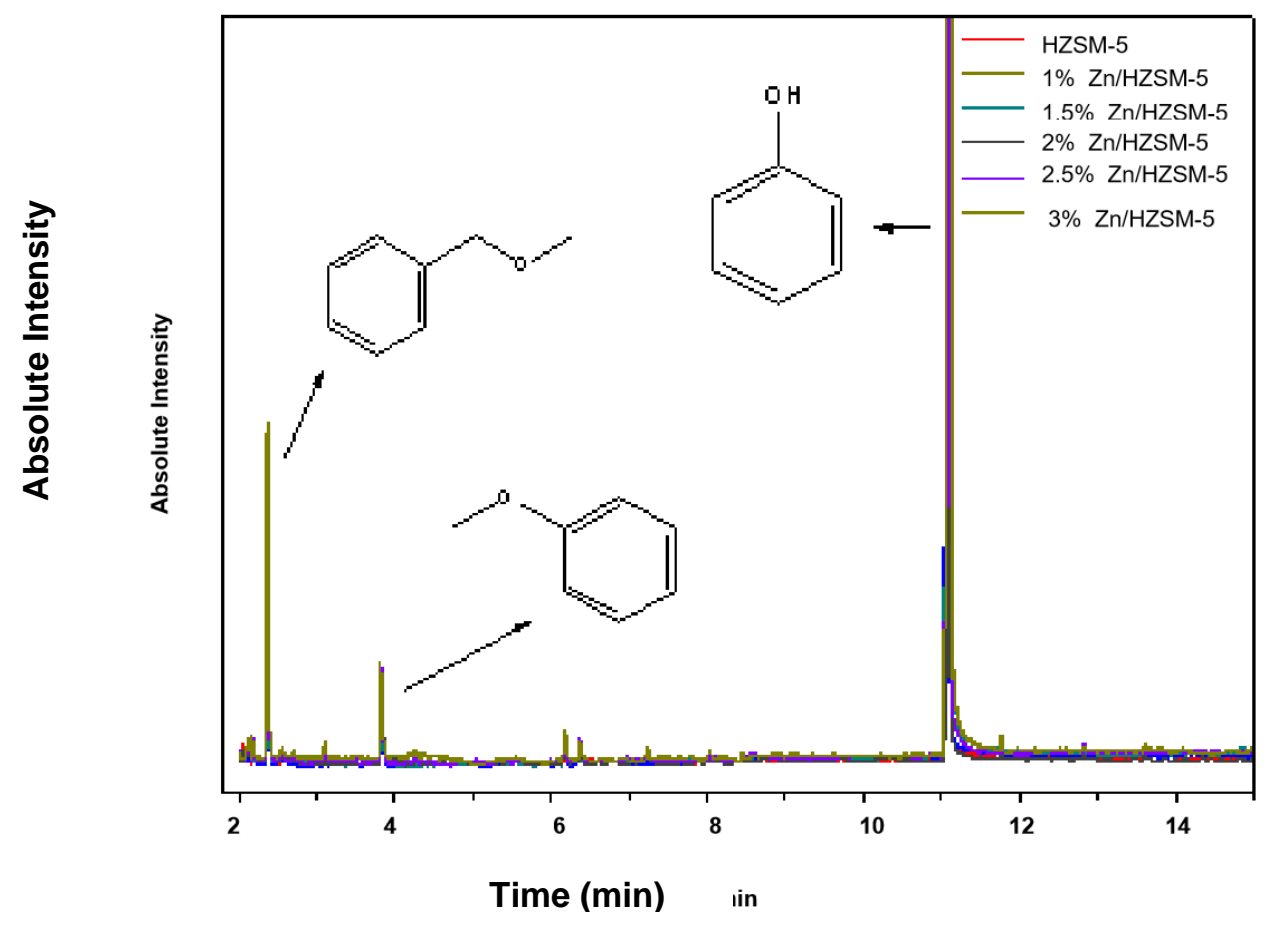




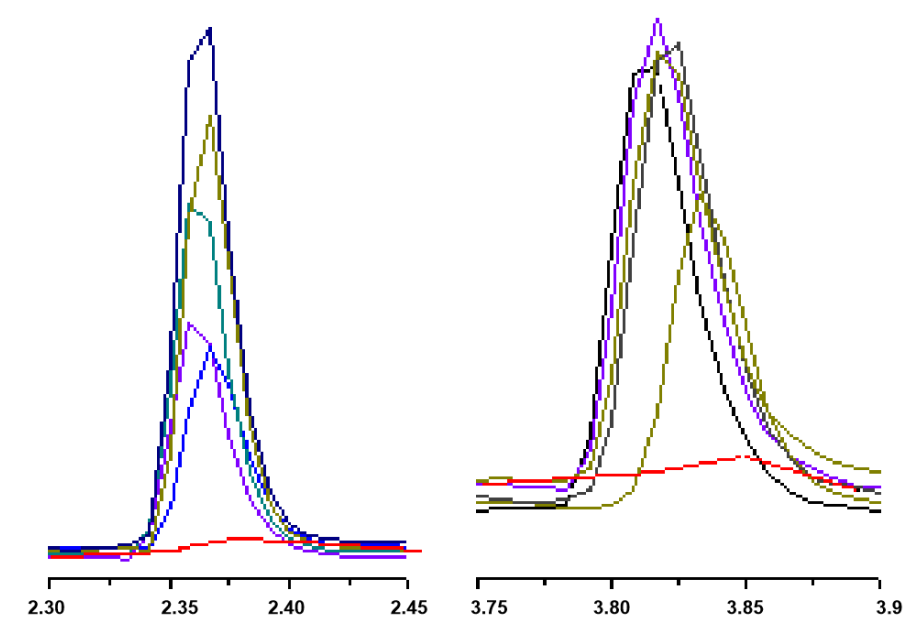

Fig. 7. GC-MS spectra for catalytic liquefaction products with Zn/HZSM-5 catalysts

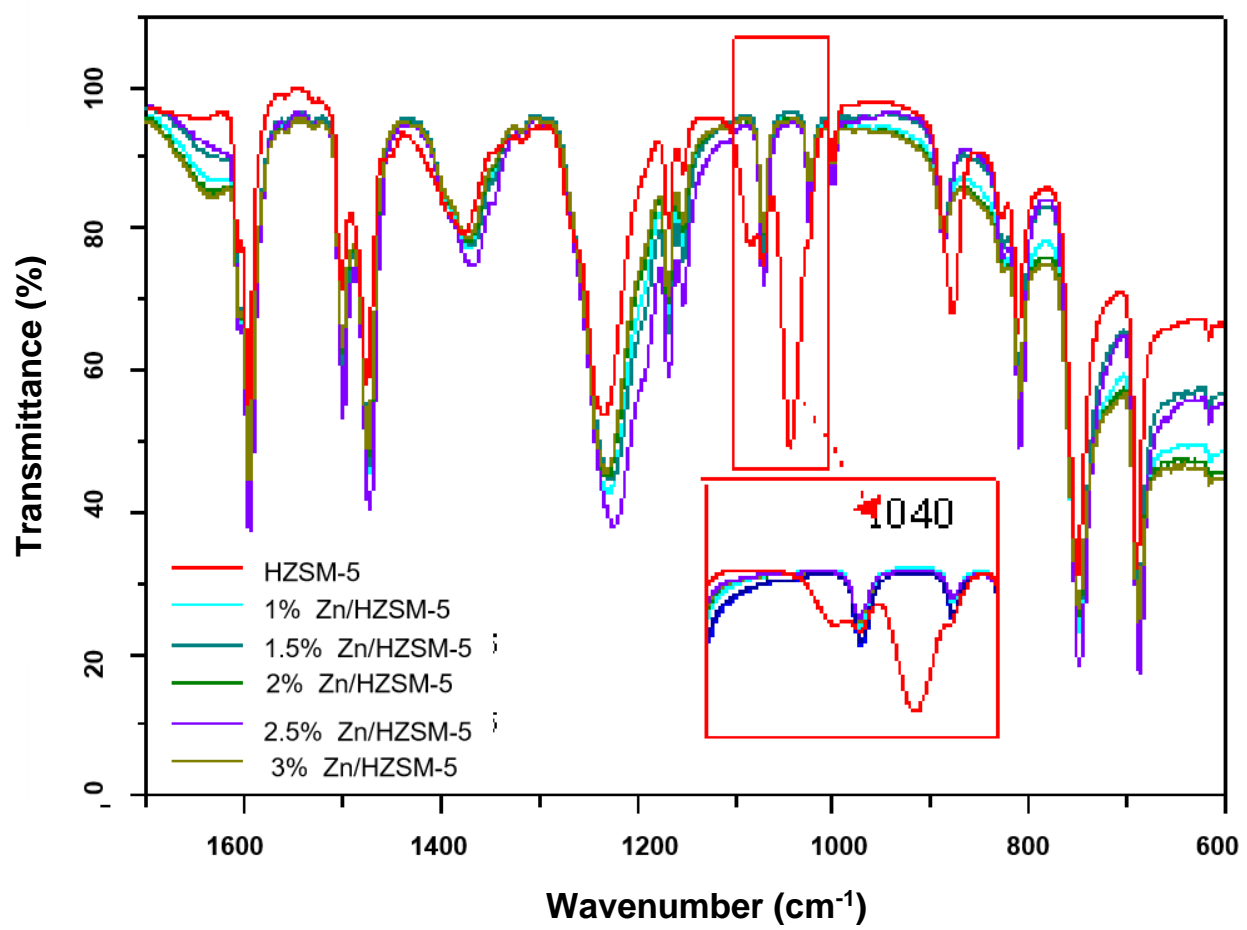

Fig. 8. FTIR spectra of liquefied product via Zn/HZSM-5 catalysts

\section{CONCLUSIONS}

The catalyst $\mathrm{Zn} / \mathrm{HZSM}-5$ was prepared using $\mathrm{Zn}^{+}$on a HZSM-5 support through conventional incipient wetness impregnation. Liquefied walnut shell was catalyzed by Zn/HZSM-5 catalysts to estimate their catalytic performance. The following conclusions were obtained through analysis:

1. The X-ray diffraction (XRD) results showed that all of the catalysts retained the original HZSM-5 crystal structure, demonstrating that $\mathrm{Zn}$ had good metal dispersion 
on catalyst surfaces. However, apart from 2\% Zn/HZSM-5, compared with the unloaded metal catalyst, the interplanar spacing increased. Particularly in the $2 \%$ $\mathrm{Zn} / \mathrm{HZSM}-5$ catalyst, the $\mathrm{Zn}$ was able to maintain an interaction balance between the $\mathrm{Zn}$ with Al framework and $\mathrm{Zn}$ with external Brønsted-Lowry acid sites on the surface of the catalyst.

2. The Brunauer-Emmett-Teller (BET) data indicated that the pore size of $2 \% \mathrm{Zn} / \mathrm{HZSM}$ 5 was the largest of the metal supported catalysts but still less than that of the unsupported. The hysteresis loop model in the adsorption-desorption isotherm illustrated that the catalyst was still in the shape of spherical particles, with a narrow pore size distribution and uniform particle size even through the interior of the catalyst loaded metal.

3. Fourier transform infrared (FTIR) analysis of the catalysts implied that with an increase in the load ratio, the amplitude of Si-O stretching vibration first decreased and then increased, while the lowest amplitude point occurred when metal was loaded at $2 \%$, which was also due to its interaction with Al and the Brønsted-Lowry acid on the catalyst surface.

4. The nuclear magnetic resonance (NMR), gas chromatography - mass spectrometry (GC-MS), and FTIR data of the liquefied product indicated that the catalysts that supported metal promoted the formation of anisole, a small-molecule ether, whereas the pure catalysts did not produce this small-molecule ether. In addition, the catalyst $2 \%$ Zn/HZSM-5 generated a high amount of small-molecule ether.

\section{ACKNOWLEDGEMENTS}

This work was supported by the Key Project of Yunnan Provincial Science Foundation (Grant No. 2014FA034), the Agricultural basic research joint project of Yunnan Province (Grant No. 2018FG001-061), the National Natural Science Foundation of China (Grant Nos. 31670599 and 31160147), and the Fundamental Research Funds for the Central Universities (Grant No. 2572017AB07).

\section{REFERENCES CITED}

Abdelsayed, V., Shekhawat, D., Poston, Jr., J. A., and Spivey, J. J. (2013). "Synthesis, characterization, and catalytic activity of Rh-based lanthanum zirconate pyrochlores for higher alcohol synthesis," Catalysis Today 207(21), 65-73. DOI:

10.1016/j.cattod.2012.07.011

Abdelsayed, V., Shekhawat, D., and Smith, M. W. (2015). "Effect of Fe and Zn promoters on Mo/HZSM-5 catalyst for methane dehydroaromatization," Fuel 139, 401-410. DOI: 10.1016/j.fuel.2014.08.064

Alzari, P. M., Spinelli, S., Mariuzza, R. A., Boulot, G., Poljak, R. J., Jarvis, J. M., and Milstein, C. (1990). "Three-dimensional structure determination of an anti-2phenyloxazolone antibody: The role of somatic mutation and heavy/light chain pairing in the maturation of an immune response," The EMBO Journal 9(12), 38073814. DOI: $10.1002 / j .1460-2075.1990 . t b 07598 . x$ 
Asadieraghi, M., and Mohd, A. W. D. W. (2015). "In-situ catalytic upgrading of biomass pyrolysis vapor: Using a cascade system of various catalysts in a multi-zone fixed bed reactor," Energy Conversion and Management 101, 151-163. DOI: 10.1016/j.enconman.2015.05.008

Batistela, V. R., Fogaça, L. Z., Fávaro, S. L., Caetano, W., Fernandes-Machado, N. R. C., and Hioka, N. (2017). "ZnO supported on zeolites: Photocatalyst design, microporosity and properties," Colloids and Surfaces A: Physicochemical and Engineering Aspects 513, 20-27. DOI: 10.1016/j.colsurfa.2016.11.023

Berry, R. S., and Smirnov, B. M. (2009). "Phase transitions in metal clusters and cluster catalysts," Journal of Physical Chemistry A 113(52), 14220-14226. DOI: 10.1021/jp9017053

Cinelli, P., Anguillesi, I., and Lazzeri, A. (2013). "Green synthesis of flexible polyurethane foams from liquefied lignin,” European Polymer Journal 49(6), 11741184. DOI: 10.1016/j.eurpolymj.2013.04.005

Cox, B. J. (2012). Depolymerization of Lignin for Biomass Processing in Ionic Liquids, Ph.D. Dissertation, University of Texas at Austin, Austin, TX, USA.

Davantès, A., Costa, D., and Lefèvre, G. (2015). "Infrared study of (poly)tungstate ions in solution and sorbed into layered double hydroxides: Vibrational calculations and in situ analysis," Journal of Physical Chemistry C 119(22), 12356-12364. DOI: 10.1021/acs.jpcc.5b01578

Fattahi, M., Behbahani, R. M., and Hamoule, T. (2016). "Synthesis promotion and product distribution for HZSM-5 and modified Zn/HZSM-5 catalysts for MTG process," Fuel 181, 248-258. DOI: 10.1016/j.fuel.2016.04.120

Fei, W., Wei, Y. X., and Guo, M. X. (2015). "Atomic layer deposition of zinc oxide on HZSM-5 template and its methanol aromatization performance," Catalysis Letters 145(3), 860-867. DOI: 10.1007/s10562-015-1496-5

Gordobil, O., Egüés, I., Llano-Ponte, R., and Labidi, J. (2014). "Physicochemical properties of PLA lignin blends," Polymer Degradation and Stability 108, 330-338. DOI: 10.1016/j.polymdegradstab.2014.01.002

Huang, S., Mahmood, N., Zhang, Y., Tymchyshyn, M., Yuan, Z., and Xu, C. C. (2017). "Reductive de-polymerization of kraft lignin with formic acid at low temperatures using inexpensive supported Ni-based catalysts," Fuel 209, 579-586. DOI: 10.1016/j.fuel.2017.08.031

Ihsan, M., Wang, H., Majid, S. R., Yang, J., Kennedy, S. J., Guo, Z., and Liu, H. K. (2016). " $\mathrm{MoO}_{2} / \mathrm{Mo}_{2} \mathrm{C} / \mathrm{C}$ spheres as anode materials for lithium ion batteries," Carbon 96, 1200-1207. DOI: 10.1016/j.carbon.2015.10.076

Isa, K. M., Abdullah, T. A. T., and Ali, U. F. M. (2018). "Hydrogen donor solvents in liquefaction of biomass: A review," Renewable and Sustainable Energy Reviews 81(Part 1), 1259-1268. DOI: 10.1016/j.rser.2017.04.006

Jackson, M. A., Compton, D. L., and Boateng, A. A. (2009). "Screening heterogeneous catalysts for the pyrolysis of lignin," Journal of Analytical and Applied Pyrolysis 85(1-2), 226-230. DOI: 10.1016/j.jaap.2008.09.016

Jaworski, J. S., Cembor, M., and Orlik, M. (2005). "Anisole as a solvent for organic electrochemistry," Journal of Electroanalytical Chemistry 582(1-2), 165-170. DOI: 10.1016/j.jelechem.2005.01.003

Kadla, J. F., and Kubo, S. (2004). "Lignin-based polymer blends: Analysis of intermolecular interactions in lignin-synthetic polymer blends," Composites Part A: Applied Science and Manufacturing 35(3), 395-400. DOI: 
10.1016/j.compositesa.2003.09.019

Kang, S., Li, X., Fan, J., and Chang, J. (2013). "Hydrothermal conversion of lignin: A review," Renewable and Sustainable Energy Reviews 27(6), 546-558. DOI: 10.1016/j.rser.2013.07.013

Kordić, B., Kovačević, M., Sloboda, T., Vidović, A., and Jović, B. (2017). "FT-IR and NIR spectroscopic investigation of hydrogen bonding in indole-ether systems," Journal of Molecular Structure 1144, 159-165. DOI: 10.1016/j.molstruc.2017.05.035

Kubo, S., and Kadla, J. F. (2005). "Lignin-based carbon fibers: Effect of synthetic polymer blending on fiber properties," Journal of Polymers and the Environment 13(2), 97-105. DOI: 10.1007/s10924-005-2941-0

Lai, Y., and Veser, G. (2016). "Zn-HZSM-5 catalysts for methane dehydroaromatization," Environmental Progress and Sustainable Energy 35(2), 334-344. DOI: 10.1002/ep.12322

Lee, W. J., Chang, K. C., and Tseng, I. M. (2011). "Properties of phenol-formaldehyde resins prepared from phenol-liquefied lignin," Journal of Applied Polymer Science 124(6), 4782-4788. DOI: 10.1002/app.35539

Li, L., Cui, X., Li, J., and Wang, J. (2015). "Synthesis of SAPO-34/ZSM-5 composite and its catalytic performance in the conversion of methanol to hydrocarbons," Journal of the Brazilian Chemical Society 26(2), 290-296. DOI: 10.5935/0103-5053.20140279

Liu, H., Yang, S., Hu, J., Shang, F., Li, Z., Xu, C., Guan, J., and Kan, Q. (2012). “A comparison study of mesoporous Mo/H-ZSM-5 and conventional Mo/H-ZSM-5 catalysts in methane non-oxidative aromatization," Fuel Processing Technology 96, 195-202. DOI: 10.1016/j.fuproc.2011.12.034

Ma, Q., Liu, Q., Li, W., Ma, L., Wang, J., Liu, M., and Zhang, Q. (2017). “Catalytic depolymerization of lignin for liquefied fuel at mild condition by rare earth metals loading on CNT," Fuel Processing Technology 161, 220-225. DOI: 10.1016/j.fuproc.2016.09.003

Mhamdi, M., Ghorbel, A., and Delahay, G. (2009). "Influence of the V + Mo/Al ratio on vanadium and molybdenum speciation and catalytic properties of V-Mo-ZSM-5 prepared by solid-state reaction," Catalysis Today 142(3-4), 239-244. DOI: 10.1016/j.cattod.2008.07.026

Mota, M. I. F., Pinto, P. C. R., Loureiro, J. M., and Rodrigues, A. E. (2016). "Recovery of vanillin and syringaldehyde from lignin oxidation: A review of separation and purification processes," Separation \& Purification Reviews 45(3), 227-259. DOI: 10.1080/15422119.2015.1070178

Mullen, C. A., Dorado, C., and Boateng, A. A. (2017). "Catalytic co-pyrolysis of switchgrass and polyethylene over HZSM-5: Catalyst deactivation and coke formation," Journal of Analytical and Applied Pyrolysis 129, 195-203. DOI: 10.1016/j.jaap.2017.11.012

Park, B., and Riedl, B. (2000). " ${ }^{13} \mathrm{C}-\mathrm{NMR}$ study on cure-accelerated phenol-formaldehyde resins with carbonates," Journal of Applied Polymer Science 77(6), 12841293. DOI: 10.1002/1097-4628(20000808)77:6<1284::AID-APP13>3.0.CO;2-K

Pradeep, A., and Chandrasekaran, G. (2006). FTIR study of Ni, $\mathrm{Cu}$ and $\mathrm{Zn}$ substituted nano-particles of $\mathrm{MgFe}_{2} \mathrm{O}_{4}$," Materials Letters 60(3), 371-374. DOI: 10.1016/j.matlet.2005.08.053

Sadrameli, S. M. (2016). "Thermal/catalytic cracking of liquid hydrocarbons for the production of olefins: A state-of-the-art review II: Catalytic cracking review," Fuel 173, 285-297. DOI: 10.1016/j.fuel.2016.01.047 
Shen, Y., Wang, F., Yang, C., and Zhang, X. (2016). "Synthesis and characterisation of hierarchically porous HZSM-5 as catalysts for the synthesis of 2,3,5-trimethyl-1,4benzoquinone," Australian Journal of Chemistry 70(6), 691-698. DOI: 10.1071/CH16392

Thakur, V. K., Thakur, M. K., Raghavan, P., and Kessler, M. R. (2014). "Progress in green polymer composites from lignin for multifunctional applications: A review," ACS Sustainable Chemistry \& Engineering 2(5), 1072-1092. DOI: $10.1021 / \mathrm{sc} 500087 \mathrm{z}$

Wang, J., Zhong, Z., Ding, K., and Xue, Z. (2016). "Catalytic fast pyrolysis of mushroom waste to upgraded bio-oil products via pre-coked modified HZSM-5 catalyst," Bioresource Technology 212, 6-10. DOI: 10.1016/j.biortech.2016.04.005

Wang, M., Bian, W., and Jiang, Z. (2017). "Estimate of carbon fiber's fracture toughness based on the small angle X-ray diffraction," Polymer Bulletin 74(10), 4143-4151. DOI: $10.1007 / \mathrm{s} 00289-017-1941-2$

Xin, Y., Qi, P., Duan, X., Lin, H., and Yuan, Y. (2013). "Enhanced performance of ZnSn/HZSM-5 catalyst for the conversion of methanol to aromatics," Catalysis Letters 143(8), 798-806. DOI: 10.1007/s10562-013-1031-5

Xu, W., Li, T., Hao, Q., Xuan, Z., Jian, W., and Zhu, T. (2015). "Effect of flue gas components on $\mathrm{Hg}^{0}$ oxidation over Fe/HZSM-5 catalyst," Industrial \& Engineering Chemistry Research 54(1), 146-152. DOI: 10.1021/ie504090m

Yin, Y., Ren, R., Liu, C., and Dong, W. (2012). "Synthesis and curing performance of the high ortho thermoplastic phenolic resin," Polymer Materials Science and Engineering 2012(4), 9-13.

Zheng, Z. F., Zou, J. C., Zhang, H. J., and Min, L. (2007). "Study on liquefaction of walnut shell in phenol and application of its resinification product as wood adhesives," Chemistry and Industry of Forest Products 27(4), 31-36. DOI: 10.3321/j.issn:0253-2417.2007.04.007

Zhou, G., Jensen, P. A., Le, D. M., Knudsen, N. O., and Jensen, A. D. (2016). "Direct upgrading of fast pyrolysis lignin vapor over the HZSM-5 catalyst," Green Chemistry 18(7), 1965-1975. DOI: 10.1039/C5GC01976A

Article submitted: January 30, 2019; Peer review completed: August 8, 2019; Revised version received and accepted: January 4, 2021; Published: January 19, 2021.

DOI: 10.15376/biores.16.1.1628-1641 